
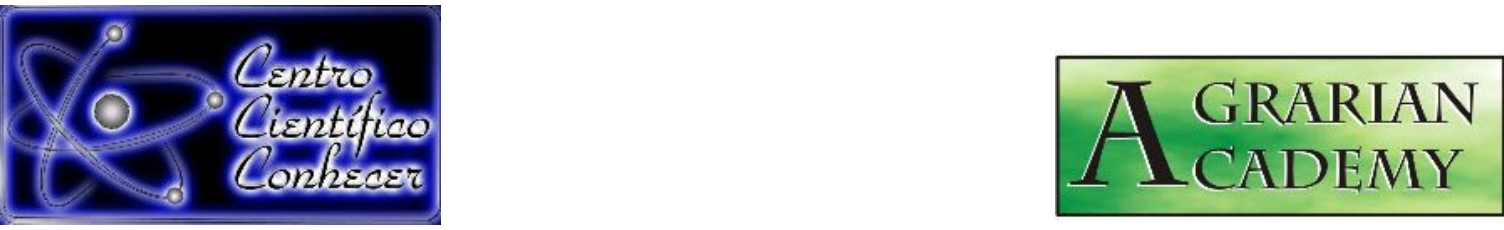

\title{
AVALIAÇÃO DA ÁREA CONSOLIDADA EM ÁREA DE PRESERVAÇÃO PERMANENTE NOS IMÓVEIS RURAIS: UM ESTUDO DE CASO EM PONTE ALTA, SANTA CATARINA
}

Moisés Savian ${ }^{1 *}$; Jonatas da Silva Campos ${ }^{2}$; Gabriela Canto Pires Santos Savian ${ }^{3}$

${ }^{1}$ Doutorando no Programa de Pós-Graduação em Produção Vegetal na Universidade do Estado de Santa Catarina (UDESC), Lages, Santa Catarina, Brasil, moisessavian@gmail.com.

${ }^{2}$ Mestre em Produção Vegetal pela Universidade do Estado de Santa Catarina (UDESC), Lages, Santa Catarina, Brasil.

3 Especialista em Meio Ambiente e Desenvolvimento pela Universidade Federal do Paraná (UFPR). Brasília, Distrito Federal, Brasil.

Recebido em: 02/06/2019 - Aprovado em: 15/06/2019 - Publicado em: 22/07/2019 DOI: 10.18677/Agrarian_Academy_2019a6

A efetivação de paisagens agrícolas sustentáveis depende da manutenção de espaços naturais em meio ao espaço alterado pelas espécies cultivadas. A legislação brasileira estabelece conceitos jurídicos que influenciam no uso do solo dos imóveis rurais, que influenciam na paisagem. Esse trabalho teve o objetivo de avaliar como a introdução do conceito de área consolidada influencia no manejo das áreas de preservação permanente (APP) em nível do imóvel rural a partir de um estudo de caso em Ponte Alta, Santa Catarina. A metodologia utilizada contou com levantamento georreferenciado a campo, processamento e análise de dados com auxílio de software de geoprocessamento. Os resultados encontrados demonstram que em média $68,3 \%$ da APP dos imóveis rurais estudados será considerada área consolidada o que levará a uma diminuição da vegetação nativa inseridos na paisagem agrícola. Conclui-se que para manter a função ambiental da área consolidada em APP, se faz necessário desenvolver sistemas produtivos mais sustentáveis, como os agroflorestais e a produção agroecológica, viabilizando a recuperação ambiental com a geração de renda.

Palavras-chave: código florestal; legislação ambiental; paisagem agrícola.

\section{EVALUATION OF THE CONSOLIDATED AREA IN A PERMANENT PRESERVATION AREA IN RURAL PROPERTIES: A CASE STUDY IN PONTE ALTA, SANTA CATARINA}

\footnotetext{
ABSTRACT

The realization of sustainable agricultural landscapes depends on the maintenance of natural spaces in the middle of the space altered by the cultivated species. Brazilian legislation establishes legal concepts that influence the use of the soil of AGRARIAN ACADEMY, Centro Científico Conhecer - Goiânia, v.6, n.11; p. 64 2019
} 
rural properties, which influence the landscape. This work had the objective of evaluating how the introduction of the concept of consolidated area influences the management of the permanent preservation areas (APP) at the rural property level from a case study in Ponte Alta, Santa Catarina. The methodology used was georeferenced to field survey, data processing and analysis with the aid of geoprocessing software. The results show that, on average, $68.3 \%$ of the APP of the rural properties studied will be considered a consolidated area, which will lead to a reduction of the native vegetation inserted in the agricultural landscape. It is concluded that to maintain the environmental function of the consolidated area in APP, it is necessary to develop more sustainable production systems, such as agroforestry and agroecological production, making environmental recovery possible with income generation.

Keywords: agricultural landscape; environmental legislation; forest code.

\section{INTRODUÇÃO}

O desenvolvimento agrícola num ambiente anteriormente natural tende a resultar em um mosaico heterogêneo de variados tipos de habitats, distribuídos na forma de manchas ao longo da paisagem (GLIESSMAN, 2001). A paisagem rural desempenha múltiplas funções, não somente a econômica, mas também funções sociais, ambientais e culturais (GALVÃO; VARETA, 2010).

No Brasil a ideia de se proteger determinadas áreas representativas dos ecossistemas naturais vem desde a criação do Código Florestal de 1934 que definia categorias de florestas protetoras, remanescentes, modelo e de rendimento, no entanto, apesar das boas intenções a legislação não funcionou devido a inércia das autoridades (BORGES et al, 2011). Em sua última atualização o Código Florestal manteve conceitos como o de área de preservação permanente (APP) e de reserva legal $(R L)$ e introduziu o de área consolidada $(A C)$, que autoriza a continuidade de atividades agrossilvipastoris, de ecoturismo e de turismo rural em áreas de preservação permanente (BRASIL, 2012).

O estabelecimento desses conceitos jurídicos possui alinhamento com a abordagem científica relacionadas as paisagens agrícolas. Gliessmann (2001) define que são três tipos básicos de componentes da paisagem agrícola: as áreas de produção agrícola, as áreas de influência humana moderada ou reduzida e as áreas naturais. O conceito de AC possibilita uma flexibilização do regime de APP fazendo com que áreas que seriam mantidas sem a ação humana passam a ser manejadas economicamente. Para De Azevedo (2013) o novo panorama estabelecido no código com relação a APP e as flexibilização na proteção visa atender os interesses de ordem socioeconômica em detrimento da proteção ambiental.

A agricultura familiar no Brasil representa mais de $84 \%$ dos estabelecimentos agropecuários do país e em Santa Catarina corresponde a $87 \%$ do total das propriedades de todo o estado (BRASIL, 2015; 2016). O Código Florestal foi alterado sob as premissas que a lei florestal gerava entraves a produção e prejudicava os pequenos produtores rurais (RORIZ; FEARNSIDE, 2015).

Nesse contexto, o objetivo desse trabalho foi avaliar de que forma a alteração da lei florestal com a introdução do conceito de área consolidada vai influenciar no manejo da APP no nível da propriedade a partir de um estudo de caso em dois imóveis rurais da agricultura familiar no município de Ponte Alta, Santa Catarina. 


\section{METODOLOGIA}

O estudo foi realizado em Ponte Alta posicionado na área central do estado de Santa Catarina, Brasil, que possui área de $569,154 \mathrm{~km}^{2}$ e população de 4.741 habitantes (IBGE, 2018). O município tem como coordenadas de referência a latitude $27^{\circ} 20^{\prime} \mathrm{S}$ e longitude $50^{\circ} 22^{\prime} \mathrm{O}$ e possui altitude média de 860 metros com clima mesotérmico úmido temperado (Cfb), segundo classificação KÖPPEN (PANDOLFO, et al., 2002). A temperatura média anual é de $15,7^{\circ} \mathrm{C}$ e precipitação acumulada por ano de 1.560,9 mm (EMBRAPA, 2012).

A economia está fortemente ligada a atividade agropecuária que representa 42,9\% do PIB municipal (IBGE, 2015). As terras estão distribuídas em 409 estabelecimentos agropecuários que ocupam $48.283,46$ hectares (IBGE, 2017). A paisagem é predominantemente composta por campos e florestas naturais e pelos reflorestamentos, especialmente do gênero Pinus, também ocorrendo, em áreas menos extensas as lavouras temporárias (SAVIAN, 2011).

Para realização dos trabalhos foram avaliados dois imóveis rurais com áreas de 7,28 e 29,62 hectares (ha) no município de Ponte Alta onde o módulo fiscal (MF) é 22 ha. A escolha dos imóveis foi realizada considerando a presença de área consolidada e buscando área com 0,1 a $1 \mathrm{MF}$ (imóvel A - 0,66 MF) e outra com entre 1,1 a 2 MF (imóvel B - 1,35 MF). O levantamento dos limites ou divisas dos imóveis, dos recursos hídricos e do uso do solo foi realizado utilizando aparelho de GPS de precisão, modelo SR-20, da marca Leica. O processamento dos dados foi realizado no software GisDataPro V 3.18.

Com os dados brutos, procedeu-se a quantificação das APPs utilizando o programa AutoCad 2015. Foram desconsideradas as APPS em declividades superiores a $45^{\circ}$ pois, conforme observou Jacovine et al. (2008), apresentam valores insignificantes quando comparadas com o total das APPs. A APP total foi classificada em duas categorias: (1) APP a ser conservada ou recuperada, que é aquela com a presença de vegetação nativa ou que a legislação exige a recuperação; e (2) APP com área consolidada, que é aquela que a lei autoriza a continuidade de atividades agrossilvipastoris. As APPs a serem mantidas ou recuperadas foram definidas a partir do artigo 61-A da Lei $n^{\circ} 12.651$ (BRASIL, 2012) o qual estabelece que para imóveis com até $1 \mathrm{MF}$ deverá ser recuperada uma faixa marginal de 5 metros (Imóvel A), e para imóveis com 1 com até $2 \mathrm{MF}$ a faixa será de 8 metros (Imóvel B). Por fim, foram elaborados os mapas e analisado os dados.

\section{RESULTADOS}

Segundo Figura 1 é possível observar que o Imóvel A foi encontrado uma nascente, quatro cursos d'água com largura inferior a dez metros e dois banhados. Para o cálculo da APP considerou um raio de 50 metros para nascentes e uma faixa de 30 metros para os rios. Com a alocação de todas as APPs formam-se três grandes áreas, sendo uma a partir da nascente e as demais pelos cursos d'águas. O uso agrícola é predominante no imóvel com $69,1 \%$ ocupada por pastagem e lavoura, seguido pela mata com $21,6 \%$ e pela área de banhado com $6,9 \%$.

No Imóvel B, de acordo com Figura 1, foram encontradas quatro nascentes, quatro cursos d'água com largura menor que 10 metros, sendo que um desses se bifurca, e três banhados. Com a alocação de todas as APPs forma-se três grandes áreas contínuas. O uso agrícola é predominante com $83,9 \%$ de campo nativo com pequenas áreas de lavouras, seguido pela mata com 11,9\% e hidrografia com 2,7\%. Os dados referentes ao uso do solo nos imóveis A e B encontram-se na Tabela 1. 
Tabela 1: Uso do solo e nos imóveis rurais A e B localizados no município de Ponte Alta, Santa Catarina.

\begin{tabular}{ccccc}
\hline Categoria & $\begin{array}{c}\text { Imóvel A } \\
\mathrm{m}^{2}\end{array}$ & $\begin{array}{c}\text { Imóvel B } \\
\mathrm{m}^{2}\end{array}$ & $\begin{array}{c}\text { \% Imóvel } \\
\mathbf{A}\end{array}$ & $\begin{array}{c}\text { \% Imóvel } \\
\mathbf{B}\end{array}$ \\
\hline Banhado & $5.011,76$ & $2.302,79$ & $6,9 \%$ & $0,8 \%$ \\
\hline $\begin{array}{c}\text { Campo e } \\
\text { Lavoura }\end{array}$ & $50.339,78$ & $248.584,27$ & $69,1 \%$ & $83,9 \%$ \\
\hline Edificações & - & $2.117,37$ & - & $0,7 \%$ \\
\hline Hidrografia & $1.746,37$ & $8.099,34$ & $2,4 \%$ & $2,7 \%$ \\
\hline Mata & $15.745,91$ & $35.140,89$ & $21,6 \%$ & $11,9 \%$ \\
\hline Área Total & $72.843,82$ & $296.244,66$ & - & -
\end{tabular}

Fonte: Elaborado pelos autores.

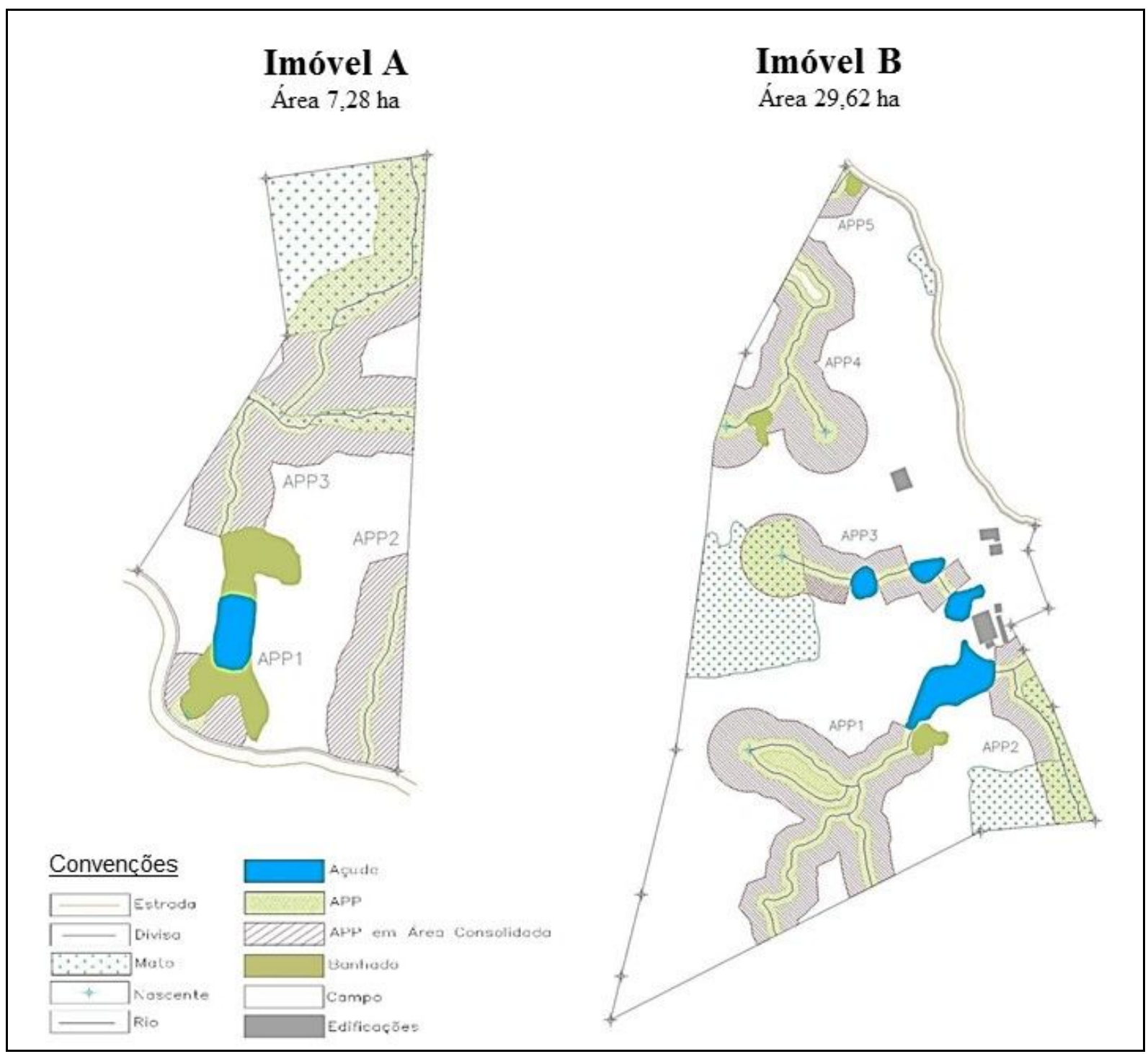

Figura 1- Croqui com alocação do uso do solo e das áreas de preservação permanente dos imóveis rurais analisados em Ponte Alta, Santa Catarina. Fonte: Elaborados pelos autores. 
Encontrou-se uma APP total de $25.972,30 \mathrm{~m}^{2}$ no imóvel A o que corresponde a $35,68 \%$ de toda área. Dessa, a maior parte $(78,16 \%)$ é considerada área consolidada e não precisará ser recuperada. No imóvel B a APP total é de $92.864,00$ $\mathrm{m}^{2}$ o que corresponde a $31,35 \%$ da área do imóvel, sendo que $58,53 \%$ é AC. $\mathrm{O}$ percentual de AC em APP por parcelas variou entre 32,22 a 90,04\%. Considerando toda a área de APP em cada imóvel o percentual médio de AC na APP nos imóveis avaliados é de 68,35, e uma área total de 7,46 ha será considerada $A C$ nos dois imóveis. A Tabela 2 apresenta os dados detalhados das parcelas de APP, áreas a serem mantidas ou recuperadas, e a APP total, bem como o percentual de AC na área total de APP.

Tabela 2: Composição das áreas de preservação permanente - APP, de acordo com o conceito de área consolidada - AC, dos imóveis rurais $A$ e $B$ localizados no município de Ponte Alta, Santa Catarina.

\begin{tabular}{|c|c|c|c|c|c|}
\hline Local & $\begin{array}{l}\text { Parcela } \\
\text { APP }\end{array}$ & $\begin{array}{c}\text { APP* }^{*} \\
\text { Manter/Recuperar }\end{array}$ & $\begin{array}{c}\mathbf{A C}^{*} \\
\text { Em APP }\end{array}$ & $\begin{array}{l}\text { APP* }^{*} \\
\text { Área Total }\end{array}$ & $\begin{array}{c}\text { \% de AC } \\
\text { na APP } \\
\text { Total }\end{array}$ \\
\hline \multirow{4}{*}{ Imóvel A } & 1 & $2.773,78$ & $1.757,12$ & $4.530,90$ & $38,78 \%$ \\
\hline & 2 & $1.535,71$ & $6.221,45$ & $7.757,16$ & $80,20 \%$ \\
\hline & 3 & $1.362,64$ & $12.321,60$ & $13.684,24$ & $90,04 \%$ \\
\hline & Total & $5.672,13$ & $20.300,17$ & $25.972,30$ & $78,16 \%$ \\
\hline \multirow{6}{*}{ Imóvel B } & 1 & $14.587,79$ & $23.537,76$ & $38.125,55$ & $61,74 \%$ \\
\hline & 2 & $7.306,73$ & $3.473,91$ & $10.780,64$ & $32,22 \%$ \\
\hline & 3 & $7.887,80$ & $7.039,67$ & $14.927,47$ & $47,16 \%$ \\
\hline & 4 & $7.910,64$ & $19.268,64$ & $27.179,28$ & $70,89 \%$ \\
\hline & 5 & 821,08 & $1.029,98$ & $1.851,06$ & $55,64 \%$ \\
\hline & Total & $38.514,04$ & $54.349,96$ & $92.864,00$ & $58,53 \%$ \\
\hline
\end{tabular}

${ }^{\star}$ Áreas apresentadas em $\mathrm{m}^{2}$.

Fonte: Elaborado pelos autores, conforme dados da pesquisa.

\section{DISCUSSÃO}

Analisando pelo conceito das paisagens agrícolas de Gliessman (2001) e pelo Código Florestal (2012) as APPs são áreas onde o ecossistema natural deveria ser mantido, no entanto quando parte dessas áreas são consideradas áreas consolidadas e é autorizado o uso agrossilvipastoris essas se efetivam com o uso antrópico, perdendo em partes suas funções ecológicas. Nos casos estudados essa perda foi de $78,16 \%$ para o imóvel A e $58,53 \%$ para o imóvel. Destaca-se uma maior presença da $A C$ no imóvel menor, o que nos casos estudados está relacionado a menor disponibilidade de terra para a agricultura familiar que consequentemente leva a uma maior necessidade de uso agrícola para o sustento da família.

Os resultados demonstram que, nos imóveis estudados, a maior parte da APP será consolidada o que permitirá a continuidade de atividades agrossilvipastoris confirmando com outras pesquisas já realizadas. Schneider et al (2016) ao estudar o município de São José dos Ausentes, Rio Grande do Sul, observaram uma redução 
na proteção da APP de 23,86\%. Borges et al. (2017) em estudo na sub-bacia do Rio São Francisco, norte do estado de Minas Gerais, concluíram que a área consolidada atingirá $95 \%$ dos produtores dessa região, refletindo em uma considerável redução de áreas protegidas ao longo de corpos d'água, afetando no controle de enchentes e de processos erosivos em bacias hidrográficas.

Portanto, faz-se necessário que sejam desenvolvidos e implantados sistemas produtivos de menor impacto ambiental e maior biodiversidade nas áreas consolidadas, como por exemplo a produção agroecológica e os sistemas agroflorestais (SAFs). Os SAFs, por exemplo, podem ajudar a proteger e alimentar a biodiversidade, mitigar as mudanças climáticas, controlar a erosão, permitir a ciclagem de nutrientes entre outros benefícios ambientais (MICCOLIS, 2017). Essa possibilidade de integrar a conservação da biodiversidade nas diferentes paisagens, aliando a geração de trabalho e renda para os agricultores tem conferido aos SAFs a possibilidade de serem uma estratégia de restauração ecológica (SAIS e DE OLIVEIRA, 2018).

A implantação de sistemas agroecológicos ou SAFs nas áreas consolidadas em APP pode viabilizar a atividade econômica melhorando as funções ambientais dessas áreas. Comparando aspectos ambientais e econômicos em áreas de produção agroecológicas e convencionais Sangalli et al (2016), observaram que o rendimento dos agricultores foi semelhante para as culturas desenvolvidas, no entanto as unidades agroecológicas apresentaram melhor fertilidade do solo e potabilidade da água.

Atualmente não existem políticas públicas específicas para o estímulo da adoção de sistemas agroflorestais na paisagem, mas várias políticas nacionais são voltadas à diversificação da produção pela agricultura familiar (SAIS e DE OLIVEIRA, 2018). As políticas voltadas a agroecologia avançaram no Brasil nos últimos anos, no entanto permanecem periféricas pois o poder de influência do agronegócio se apresenta como um obstáculo para implantação de uma política de agroecologia capaz de reorientar as políticas de desenvolvimento rural (SCHIMITT, 2017). Para estimulo da implantação desses sistemas produtivos em APP faz-se necessário o aprimoramento e o desenvolvimento de políticas públicas que tenham a finalidade consolidar paisagens sustentáveis.

\section{CONCLUSÃO}

Os imóveis rurais apresentam a maior parte de sua APP composta por AC, o que remete a necessidade de implantação de sistemas produtivos sustentáveis nessas áreas que articulem a produção econômica com a conservação ambiental. $O$ imóvel A apresentou um percentual de AC na APP superior ao Imóvel B o que nos casos estudados está ligado a menor disponibilidade de terra para o agricultor familiar que consequentemente leva a uma maior necessidade de uso agrícola e uma maior pressão sob os recursos naturais.

\section{REFERÊNCIAS}

BORGES, L. A.C.; REZENDE, J. L. P.; PEREIRA, J. A. A.; COELHO JUNIOR, L. M.; DE BARROS, D.A. Áreas de Preservação Permanente na legislação ambiental

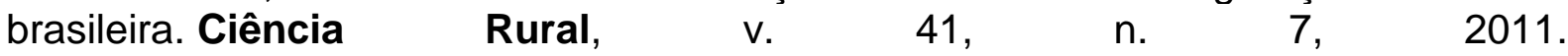
<http://submission.scielo.br/index.php/cr/article/viewFile/39118/4803>. http://dx.doi.org/10.1590/S0103-84782011000700016 
BORGES, L. A. C.; MORAS FILHO, L. O.; MARQUES, R. T.; DA SILVA, C. C.; SILVA, L. G. P. et al. A influência do tamanho do imóvel rural sobre as áreas de preservação permanente de corpos d'água. Caminhos de Geografia, v. 18, n. 64, p. 444-453, $2017 . \quad<h t t p: / / w w w . s e e r . u f u . b r / i n d e x . p h p / c a m i n h o s d e$ geografia/article/view/40953>

BRASIL. Lei Federal o 12.651, de 25 de Maio de 2012. Dispõe sobre a proteção da vegetação nativa; altera as Leis oㅡ 6.938, de 31 de agosto de 1981, ํo 9.393, de 19 de dezembro de 1996, e o 11.428, de 22 de dezembro de 2006; revoga as Leis no 4.771, de 15 de setembro de 1965, e o 7.754, de 14 de abril de 1989, e a Medida Provisória ํo 2.166-67, de 24 de agosto de 2001; e dá outras providências. Brasília, 2012.

BRASIL, Ministério do Desenvolvimento Agrário - Secretaria Especial de Agricultura Familiar e Desenvolvimento Rural. A força da Agricultura Familiar. Brasília, 2015. Disponível em: http://www.mda.gov.br/sitemda/noticias/for\%C3\%A7a-da-agriculturafamiliar. Acesso em: 18 set.2017.

BRASIL, Ministério do Desenvolvimento Agrário - Secretaria Especial de Agricultura Familiar e Desenvolvimento Rural. Aqui tem Agricultura Familiar - Santa Catarina. Áudio. Brasília, 2016. Disponível em: http://www.mda.gov.br/sitemda/radio-mda/aqui-tem-agricultura-familiar-santacatarina. Acesso em: 18 set. 2017.

DE AZEVEDO, R. E. S. O novo Código Florestal e a flexibilização das intervenções excepcionais em Áreas de Preservação Permanente. Revista Direito Ambiental e Sociedade, v. 3, n. 1, 2013.

EMBRAPA. Atlas climático da região Sul do Brasil: estados do Paraná, Santa Catarina e Rio Grande do Sul. Brasília: Embrapa, 2012. 334 p.

GALVÃO, M.; DEVY-VARETA, N. A multifuncionalidade das paisagens rurais: uma ferramenta para o desenvolvimento. Cadernos: Curso de Doutoramento em Geografia, n. ㅇ 2, 2010, p. 61-86, 2010. https://wp.ufpel.edu.br/leaa/files/2015/03/amultifuncionalidade-das-paisagens-rurais-uma-ferramenta-para-odesenovolvimento.pdf

GLIESSMAN, S. Agroecologia: processos ecológicos em agricultura sustentável. 2. Ed. Porto Alegre: Ed. UFRGS, 2001. 654 p.

IBGE. Produto Interno Bruto dos Municípios. Rio de Janeiro: IBGE, 2015. Disponível em: < https://cidades.ibge.gov.br/brasil/sc/ponte-alta/pesquisa/38/46996>. Acesso em: 10 jan. 2018.

IBGE. Censo Agropecuário 2017 - Dados Preliminares. Disponível em: https://cidades.ibge.gov.br/brasil/sc/ponte-alta/pesquisa/24/76693. Acesso em: 18 jun. 2019.

IBGE. População Estimada 2018. Disponível em: 
https://cidades.ibge.gov.br/brasil/sc/ponte-alta/panorama. Acesso em: 18 jun. 2019.

JACOVINE, L. A. G.; CORREA, J. B. L.; DA SILVA, M. L.; VALVERDE, S. R; FERNANDES FILHO, E. I. et al. Quantificação das Áreas de Preservação Permanente e de Reserva Legal em propriedades da bacia do Rio Pomba-MG. Revista Árvore, v.32, n.2, p.269-278, 2008. http://www.scielo.br/scielo.php? script=sci_arttext\&pid=S0100-67622008000200010. http://dx.doi.org/10.1590/S010067622008000200010

MICCOLIS, A.; PENEIREIRO, F. M.; MARQUES, H. R.; VIEIRA, D. L. M.; ARCOVERDE, M. F. et al. Restauração Ecológica com Sistemas Agroflorestais: como conciliar conservação com produção. Opções para o Cerrado e Caatinga. Brasília: Instituto Sociedade, População e Natureza - ISPN/Centro Internacional de Pesquisa Agroflorestal - ICRAF, 2017. ISBN: 978-85-63288-18-9, 266 p.

PANDOLFO, C.; BRAGA, H. J.; SILVA JR, V. P. da; MASSIGNAM, A. M., PEREIRA, E. S.; THOMÉ, V. M. R.; VALCI, F.V. Atlas climatológico do Estado de Santa Catarina. Florianópolis: Epagri, 2002. CD-Rom.

RORIZ, P. A. C.; FEARNSIDE, P. M.. A construção do Código Florestal Brasileiro e as diferentes perspectivas para a proteção das florestas. Novos Cadernos NAEA, v. 18, n. 2, $2015 . \quad$ <https://periodicos.ufpa.br/index.php/ncn/article/view/1866>. http://dx.doi.org/10.5801/ncn.v18i2.1866

SAIS, A. C.; DE OLIVEIRA, R. E. Distribuição de Sistemas Agroflorestais no Estado De São Paulo: apontamentos para restauração florestal e produção sustentável. REDES: Revista do Desenvolvimento Regional, v. 23, n. 1, p. 111132, 2018. <https://dialnet.unirioja.es/servlet/ articulo?codigo=6354646>. http://dx.doi.org/10.17058/redes.v23i1.8735

SANGALLI, A. R.; RECALDE, K. M. G,; DA SILVA, L. F.; PADOVAN, M. P. Aspectos ambientais e socioeconômicos em unidades de produção sob bases agroecológicas e convencionais no Assentamento Pedro Ramalho, em Mato Grosso do Sul. Revista Brasileira de Agroecologia, [S.I.], v. 1, n. 1, mar. 2016. ISSN 1980-9735. $<$ ttp://revistas.aba-

agroecologia.org.br/index.php/rbagroecologia/article/view/13406>.

SAVIAN, M. A sucessão geracional na agricultura familiar de Ponte Alta-SC. 2011.102 f. Dissertação (Mestrado em Geografia) - Universidade Federal de Santa Catarina, Florianópolis. 2011.

SCHIMITT, C.; NIERDELE, P. ; AVILA, M.; SABOURIN, E.;PETERSEN, P. et al, La experiencia brasileña de construcción de políticas públicas en favor de la Agroecología. IN: SABOURIN, Eric; NIEDERLE, Paulo André. Políticas públicas a favor de la agroecología en América Latina y el Caribe. 2017. $<$ https://www.lume.ufrgs.br/bitstream/handle/10183/186167/ 001083555.pdf? sequence $=1$ \&isAllowed $=\mathrm{y}>$.

SCHNEIDER, V. E.; MAGRIN, T. B.; CEMIN, G.; DOS SANTOS, G. M. Análise da situação ambiental das Áreas de Preservação Permanente no município de São 
José dos Ausentes-RS. Scientia cum Industria, v. 4, n. 2, p. 125-130, 2016. http://www.ucs.br/etc/revistas/index.php/scientiacumindustria/article/view/4525. http://dx.doi.org/10.18226/23185279.v4iss2p125. 